\title{
LARGEST NORMAL NEIGHBORHOODS
}

\author{
V. OZOLS
}

\begin{abstract}
It is well known that the largest normal neighborhood of a point in a compact Riemannian manifold is a Euclidean cell, that is, homeomorphic to the open unit ball. In this paper it is proved that this normal neighborhood is in fact $C^{\infty}$ diffeomorphic to the open unit ball. The method is to paste together a sequence of $C^{\infty}$ radial dilations which combine to engulf an open ball or all of $\mathbf{R}^{n}$.
\end{abstract}

Let $M$ be a compact $C^{\infty}$ Riemannian manifold of dimension $n$. For each $p \in M$ let $\tilde{C}(p)$ be the cut locus of $p$ in the tangent space $T_{p} M$. Then $\tilde{C}(p)$ is homeomorphic to the $(n-1)$-sphere $S^{n-1}$, and the bounded component $\tilde{E}_{p}$ of $T_{p} M \backslash \tilde{C}(p)$ is homeomorphic to an open $n$-ball $B^{n}$ in $\mathbf{R}^{n}$ (see [1]). It is not true in general (even when $n=2$ ) that $\tilde{C}(p)$ is a smooth manifold, in which case it cannot be diffeomorphic to $S^{n-1}$. It is natural to ask, therefore, whether $\tilde{E}_{p}$ is diffeomorphic to $B^{n}$. When $n \neq 4$, it is known [2], [3], [4] that any open set in $\mathbf{R}^{n}$ which is homeomorphic to $B^{n}$ is $C^{\infty}$ diffeomorphic to $B^{n}$. The method of proof is not elementary, however, and the result is so far not known if $n=4$. On the other hand, the sets $\tilde{E}_{p}$ encountered in Riemannian geometry have the additional property that they are star-shaped about $0 \in T_{p} M$. This property makes them more tractable, and allows us to give an elementary proof that every $\tilde{E}_{p}$ is $C^{\infty}$ diffeomorphic to $B^{n}$ with no restriction on the dimension. In fact, we will construct a diffeomorphism (a kind of "radial engulfing") which preserves directions (i.e. preserves the rays emanating from the origin).

Let $\|\cdots\|$ be the Euclidean norm in $\mathbf{R}^{n}$, and for each $r>0$ let $S_{r}$ $=\left\{x \in \mathbf{R}^{n} \mid\|x\|=r\right\}$ and $B_{r}=\left\{x \in \mathbf{R}^{n} \mid\|x\|<r\right\}$. Suppose $\mu: S_{1} \rightarrow \mathbf{R}^{1}$ is a continuous function with $\mu(\theta)>0$ for all $\theta \in S_{1}$, and define the open set $U_{\mu} \subset \mathbf{R}^{n}$ by

$$
U_{\mu}=\left\{x \in \mathbf{R}^{n} \mid x=0 \text { or } 0<\|x\|<\mu(x /\|x\|)\right\} .
$$

Lemma. If $U \subset \mathbf{R}^{n}$ is defined by a continuous function $\mu: S_{1} \rightarrow \mathbf{R}^{1}$ as above, then there is a $C^{\infty}$ diffeomorphism $h: U \rightarrow B_{1}$ which preserves directions.

Proof. Let $\mu: S_{1} \rightarrow \mathbf{R}^{1}$ be the defining function of $U$, and let $r=\inf \{\mu(\theta) \mid$

Received by the editors March 14, 1976.

AMS (MOS) subject classifications (1970). Primary 53C20; Secondary 57A50, 57C30.

Key words and phrases. Cut locus, normal neighborhood, engulfing. 
$\left.\theta \in S_{1}\right\}, R=\sup \left\{\mu(\theta) \mid \theta \in S_{1}\right\}$. Let $0<r_{0}<r$ be fixed, and choose a sequence $\left\{\varepsilon_{i}\right\}_{i=1}^{\infty}$ such that: (i) $\varepsilon_{i}>0$, (ii) $\varepsilon_{i}>\varepsilon_{i+1}$, (iii) $\varepsilon_{1}<r-r_{0}$, (iv) $\lim _{i \rightarrow \infty} \varepsilon_{i}=0$. Let $\delta_{i}=\varepsilon_{i}-\varepsilon_{i+1}$, so that $0<\delta_{i}<\varepsilon_{i}$ and $\lim \delta_{i}=0$. Define continuous functions $\tilde{\mu}_{i}: S_{1} \rightarrow \mathbf{R}^{1}$ by $\tilde{\mu}_{i}=\mu-\varepsilon_{i}+\delta_{i} / 2$. Thus, $\tilde{\mu}_{i}(\theta)$ is the midpoint between $\mu(\theta)-\varepsilon_{i}$ and $\mu(\theta)-\varepsilon_{i+1}$. For each $i$, let $\mu_{i}: S_{1} \rightarrow \mathbf{R}^{1}$ be a $C^{\infty}$ function such that $\left|\mu_{i}(\theta)-\tilde{\mu}_{i}(\theta)\right|<\delta_{i} / 4$ for all $\theta \in S_{1}$. Then $\left\{\mu_{i}\right\}$ satisfies: (a) $r_{0}<\mu_{1}(\theta)<\mu_{2}(\theta)<\cdots$; (b) $\mu_{i+1}(\theta)-\mu_{i}(\theta)>\delta_{i} / 4+\delta_{i+1} / 4$ $=\left(\varepsilon_{i}-\varepsilon_{i+2}\right) / 4>0$; (c) $\mu_{i} \rightarrow \mu$ uniformly on $S_{1}$. For each $i$, let $U_{i}=U_{\mu_{i}}$ be the set defined by $\mu_{i}$. Then all the sets $U_{i}$ satisfy $\bar{U}_{i} \subset U_{i+1}$ and $U=\cup_{i=1}^{\infty} U_{i}$. Let $\mu_{0}: S_{1} \rightarrow \mathbf{R}^{1}$ be the constant function $\mu_{0} \equiv r_{0}$, and $U_{0}=B_{r_{0}}$ the set it defines. Let $r_{i}=\inf \left\{\mu_{i}(\theta) \mid \theta \in S_{1}\right\}, R_{i}=\sup \left\{\mu_{i}(\theta) \mid \theta \in S_{1}\right\}$. Then $r_{0}<r_{1}$ $<\cdots$, and $R_{0}<R_{1}<\cdots$. Let $A_{1}=R_{1}$ and define inductively $A_{i+1}$ $=R_{i+1}\left(A_{i} / r_{i}\right)$. Then

$$
A_{i+1} / R_{i+1}=A_{i} / r_{i} \geqslant A_{i} / R_{i}=A_{i-1} / r_{i-1} \geqslant \cdots \geqslant A_{1} / R_{1}=1 .
$$

Let $\alpha: \mathbf{R}^{1} \rightarrow \mathbf{R}^{1}$ be a $C^{\infty}$ function such that: (i) $\alpha(t)=0$ if $t \leqslant 0, \alpha(t)=1$ if $t$ $\geqslant 1$; (ii) $\alpha^{\prime}(t)>0$ if $t \in(0,1)$. Introduce polar coordinates $(\theta, r)$ in $\mathbf{R}^{n}$, where $\theta \in S_{1}$ and $r \geqslant 0$. When $r>0$ and $\theta$ is restricted to a coordinate patch on $S_{1}$, then $(\theta, r)$ is a coordinate patch on $\mathbf{R}^{n} \backslash\{0\}$. Define a sequence $\left\{\eta_{i}\right\}_{i=0}^{\infty}$ of maps $\eta_{i}: \mathbf{R}^{n} \rightarrow \mathbf{R}^{1}$ inductively by: $\eta_{0} \equiv 1$, and

$$
\eta_{i}(\theta, r)=\left\{\begin{array}{r}
1 \quad \text { if } r=0, \\
\left(1-\alpha\left(\frac{r-\mu_{i-1}(\theta)}{\mu_{i}(\theta)-\mu_{i-1}(\theta)}\right)\right) \eta_{i-1}(\theta, r) \\
+\alpha\left(\frac{r-\mu_{i-1}(\theta)}{\mu_{i}(\theta)-\mu_{i-1}(\theta)}\right) \frac{A_{i}}{\mu_{i}(\theta)} \quad \text { if } r \neq 0 .
\end{array}\right.
$$

Note that for $0<r<\mu_{i-1}(\theta)$ (i.e. $\left.(\theta, r) \in \bar{U}_{i-1}\right)$, we have $\eta_{i}(\theta, r)=\eta_{i-1}(\theta, r)$; and for $r \geqslant \mu_{i}(\theta), \eta_{i}(\theta, r)=A_{i} / \mu_{i}(\theta)$. In particular, all $\eta_{i}=1$ when $0 \leqslant r$ $\leqslant r_{0}=R_{0}$. Therefore, each $\eta_{i}: U \rightarrow \mathbf{R}^{1}$ is a $C^{\infty}$ function. Moreover, if $(\theta, r) \in U_{i} \backslash U_{i-1}$, then $\eta_{i-1}(\theta, r)=A_{i-1} / \mu_{i-1}(\theta)$; and

$$
A_{i} / \mu_{i}(\theta) \geqslant A_{i} / R_{i}=A_{i-1} / r_{i-1} \geqslant A_{i-1} / \mu_{i-1}(\theta) .
$$

Thus, along each ray $r \mapsto(\theta, r)$ ( $\theta$ fixed), $\eta_{i}$ is nondecreasing in each interval $\left[\mu_{i-1}(\theta), \mu_{i}(\theta)\right]$; hence nondecreasing along the entire ray emanating from 0 . Moreover, $\eta_{i}(\theta, 0)=1$ so $\eta_{i} \geqslant 1$ on $U$. Define maps $f_{i}: U \rightarrow \mathbf{R}^{n}$ by $f_{i}(\theta, r)$ $=\left(\theta, \eta_{i}(\theta, r) r\right)$ (i.e. $\left.f_{i}(x)=\eta_{i}(x) \cdot x\right)$. These are $C^{\infty}$ maps and $f_{i} \mid \bar{U}_{i-1}$ $=f_{i-1} \mid \bar{U}_{i-1}$. Let $\eta(x)=\lim _{i \rightarrow \infty} \eta_{i}(x)$ and $f(x)=\lim _{i \rightarrow \infty} f_{i}(x)$. Since $\bar{U}_{i-1}$ $\subset U_{i}, f\left|U_{i}=f_{i}\right| U_{i}$, and $\eta\left|U_{i}=\eta_{i}\right| U_{i}$, it follows that $\eta$ and $f$ are $C^{\infty}$ maps on $U$. Note that the inductive definitions above make sense for all $x \in \mathbf{R}^{n}$, but one cannot expect $\eta$ or $f$ to be differentiable on $\partial U=\bar{U} \backslash U$ even though $\eta_{i}$ and $f_{i}$ are. Let $\theta=\left(\theta_{1}, \ldots, \theta_{n-1}\right)$ be a coordinate chart on $S_{1}$, and compute $D f$ in terms of the local coordinates $\left(\theta_{1}, \ldots, \theta_{n-1}, r\right)$ in $U \backslash\{0\}$. It is easily seen that in $U_{i} \backslash\{0\}$, we have 


$$
D f=\left(\begin{array}{c|c}
I_{n-1} & 0 \\
\hline * & r \partial \eta_{i} / \partial r+\eta_{i}
\end{array}\right)
$$

where $I_{n-1}$ is the $(n-1) \times(n-1)$ identity matrix. Thus, $\operatorname{det}(D f)=r \partial \eta_{i} / \partial r$ $+\eta_{i}$. But $\eta_{i}$ is nondecreasing in the $r$-direction so $\partial \eta_{i} / \partial r \geqslant 0$; and since $r \geqslant 0, \eta_{i} \geqslant 1$, it follows that $\operatorname{det}(D f)>0$ in $U \backslash\{0\}$. In a neighborhood of $0, f=\mathrm{id}$, so $f$ is nonsingular on $U$. Since $f$ preserves the rays $r \mapsto(\theta, r)$, and $\eta_{i} \geqslant 1$, it follows that $f$ is one-to-one on $U$. Since $A_{i+1}=R_{i+1} A_{i} / r_{i}$ and $R_{i+1} / r_{i} \geqslant 1$, we have $A_{1} \leqslant A_{2} \leqslant \cdots$. Thus, either $\lim A_{i}=+\infty \operatorname{or} \lim A_{i}$ $=A<+\infty$. For each $\theta \in S_{1}$,

$$
\begin{aligned}
\lim _{t \rightarrow \mu_{i}(\theta)^{-}} f(\theta, t) & =\lim f_{i}(\theta, t)=\lim \left(\theta, \eta_{i}(\theta, t) t\right) \\
& =\left(\theta, A_{i} \mu_{i}(\theta) / \mu_{i}(\theta)\right)=\left(\theta, A_{i}\right)
\end{aligned}
$$

(the limit is taken through values $t<\mu_{i}(\theta)$ ). Consequently, $f\left(U_{i}\right)=B_{A_{i}}$ for each $i=1,2, \ldots$. Therefore, either $f(U)=\mathbf{R}^{n}$ or $f(U)=B_{A}, A<+\infty$. It is easy to construct direction preserving $C^{\infty}$ diffeomorphisms $\mathbf{R}^{n} \rightarrow B_{1}, B_{A}$ $\rightarrow B_{1}$, so the proof is complete. Q.E.D.

In the case of a compact Riemannian manifold, the sets $\tilde{E}_{p}$ are all defined by positive continuous functions $\mu: S_{1} \rightarrow \mathbf{R}^{1}$, so by the lemma:

THEOREM. Let $M$ be a compact $C^{\infty}$ Riemannian manifold, $p \in M$ any point, $\tilde{C}(p)$ the cut locus of $p$ in $T_{p} M$, and $\tilde{E}_{p}$ the bounded component of $T_{p} M \backslash \tilde{C}(p)$. Then there is a direction preserving $C^{\infty}$ diffeomorphism $\tilde{E}_{p} \rightarrow B_{1}$.

Since $\exp _{p} \tilde{E}_{p}$ is the largest normal neighborhood of $p$ in $M$, this theorem shows that $\exp _{p} \tilde{E}_{p}$ is diffeomorphic to the open unit ball.

\section{REFERENCES}

1. S. Kobayashi and K. Nomizu, Foundations of differential geometry, Vol. 2, Interscience, New York, 1969. MR 38 \#6501.

2. E. E. Moise, Affine structures in 3-manifolds. V. The triangulation theorem and Hauptvermutung, Ann. of Math. (2) 56 (1952), 96-114. MR 14, 72.

3. J. Munkres, Obstructions to the smoothing of piecewise-differentiable homeomorphisms, Ann. of Math. (2) 72 (1960), 521-554. MR 22 \# 12534.

4. J. R. Stallings, The piecewise-linear structure of Euclidean space, Proc. Cambridge Philos. Soc. 58 (1962), 481-488. MR 26 \#6945.

Department of Mathematics, University of Washington, Seattle, Washington 98195 